\title{
The Synthesis of Novel Annelated 2-Oxopiperazines by the Interaction Methyl (3-Oxopiperazin-2-Ylidene) Acetate with an $\mathrm{N}$-Arylmaleimides
}

\author{
Svetlana Mikhajlovna Medvedeva ${ }^{*}$ and Khidmet Safarovich Shikhaliev \\ Faculty of chemistry, Voronezh state university, Voronezh 394006, Russia
}

\begin{abstract}
It is studied that the interaction of methyl (3-oxopiperazin-2-ylidene) acetate with an $\mathrm{N}$-arylmaleimides. It is established that methyl (3-oxo-piperazine-2-ylidene) acetate is reacted with $N$-arylmaleimides in boiling methanol in the presence of catalytic amounts of acetic acid as the $C$ - $N$-dinucleophile to form not previously described methyl-1, 6-dioxo-8-[(arylamino)carbonyl]-1, 3, 4, 6, 7, 8-hexahydro-2H-pyrido [1, 2-a] pyrazine-9-carboxylates. An excess of acid (in a mixture of methanol and acetic acid in the ratio 1:1) occurs 1, 3-dipolar cycloaddition of the $N$-arylmaleimides toward tautomeric form methyl (3-oxo-piperazine-2-ylidene) acetate methyl (3-oxo-3, 4, 5, 6-tetrahydropyrazine-2-yl) acetate with the formation of new heterocyclic system-methyl-2-aryl-1, 3, 8-trioxodecahydro-1H-pyrrolo [3', 4':4, 5] pyrrolo [1, 2-a] pyrazine-9-carboxylates.
\end{abstract}

Key words: 2-oxopiperazine, methyl (3-oxo-piperazine-2-ylidene) acetate, $N$-arylmaleimide, pyrrolo[3', 4':4, 5] pyrrolo[1, 2-a] pyrazine, pyrido [1, 2-a] pyrazine.

\section{Introduction}

The 2-oxopiperazine fragment is an important pharmacophore which is found in a large number of biologically active molecules. Oxopiperazines are amongst the most important scaffolds in today's drug discovery industries. Due to the high number of positive hits encountered in biological screens with this heterocycle and its congeners, the substituted oxopiperazine is widely recognized as a "privileged scaffold" in medicinal chemistry [1]. Fragment of 2-oxopiperazine is a composite element natural substances of various structural complexity and biological activity [2-5]. It should be noted that in the structure many alkaloids (e.g., agelastatine A, marcfortine B, Phakellin group) 2-oxopiperazine moiety condensed to various cycles [3-5]. To date it was developed many effective methods for synthesis of highly substituted, including chiral

*Corresponding author: Svetlana Mikhajlovna Medvedeva, Ph.D., associate professor, research field: chemistry of heterocyclic compounds. E-mail: SMMedvedeva@Rambler.Ru. 2-oxopiperazines [6-9], while only a small number of them affords 2-oxopiperazin annelated by the bond $\mathrm{C}(3)-\mathrm{N}(4)$ [7-9]. In this connection, it is of interest that the synthesis of a novel polyheterocyclic structures containing 2-oxopiperazine moiety were annelated by the C(3)-N(4) bond to different cycles. The purpose of this paper is to provide novel annelated heterocycles among 2-oxopiperazine.

\section{Experiment}

\subsection{Characterization}

Control over the individuality of the reagents and the obtained compounds, as well as the progress of the reaction was monitored by TLC on Silufol UV-254. As eluent was used chloroform; the manifestation of chromatograms was in UV light and iodine vapor. ${ }^{1} \mathrm{H}$ NMR spectra were recorded on the instrument Bruker AC-300 (300 MHz); internal standard-TMS, solvents-dimethylsulfoxide, deuterium. Mass-spectra were removed on the device LKB 9000 with the input of the substance directly in the ionizing source, the 
energy of ionizing electrons $70 \mathrm{EV}$. All solvents and chemicals used in this work were analytical grade and used without purification.

2.2. General Method for the Synthesis of Methyl-2-Aryl - 1, 3, 8 - Trioxodecahydro-1H-Pyrrolo [3', 4':4, 5] Pyrrolo [1, 2-a] Pyrazine-9-Carboxylates $3 a, b$

A mixture of $0.85 \mathrm{~g}(0.5 \mathrm{mmol})$ of 2-oxopiperazine 1 and $0.51 \mathrm{mmol}$ of the corresponding $\mathrm{N}$-arylmaleimide in a mixture of $5 \mathrm{~mL}$ of methanol and $5 \mathrm{~mL}$ of acetic acid are refluxed for $10 \mathrm{~h}$ (control-TLC). The precipitate formed is filtered off after cooling, washed with ethanol. For removal of impurities recyclization products recrystallized twice from dimethylformamide. Received compounds $3 \mathrm{a}, \mathrm{b}$ :

Methyl-2-(4-methylphenyl)-1,

3 , 8-trioxodecahydro-1H-pyrrolo [3', 4':4, 5] pyrrolo [1, 2-a] pyrazine-9-carboxylate 3a. Yield $65 \%, \mathrm{mp}$ 180-181 ${ }^{\circ} \mathrm{C}$. MS (EI, $70 \mathrm{ev)} \mathrm{m/z, \% :357} \mathrm{(8,} \mathrm{M} \mathrm{+);} 343$ (25); 326 (6); 236 (63); 218 (100); 191 (24). ${ }^{1} \mathrm{H}$ NMR (300 MHz, ДMSO-d $\left.d_{6}\right) \delta: 2.42\left(3 \mathrm{H}, \mathrm{s}, \mathrm{CH}_{3}\right) ; 2.53-2.59$ $(1 \mathrm{H}, \mathrm{m}, \mathrm{CH}) ; 2.90-2.98(1 \mathrm{H}, \mathrm{m}, \mathrm{CH}) ; 3.22-3.26(2 \mathrm{H}$, $\left.\mathrm{m}, \mathrm{CH}_{2} \mathrm{~N}\right) ; 3.40-3.44(1 \mathrm{H}, \mathrm{m}, \mathrm{CH}) ; 3.46-3.50(1 \mathrm{H}, \mathrm{m}$, $\left.\mathrm{CH}_{2} \mathrm{NH}\right) ; 3.62\left(3 \mathrm{H}, \mathrm{s}, \mathrm{CH}_{3} \mathrm{O}\right) ; 3.72-3.74(1 \mathrm{H}, \mathrm{m}$, $\left.\mathrm{CH}_{2} \mathrm{NH}\right) ; 4.46(1 \mathrm{H}, \mathrm{d}, \mathrm{J}=6.8, \mathrm{CH}) ; 7.08(2 \mathrm{H}, \mathrm{d}, \mathrm{J}=8.6$; H-3,5 Ar); 7.19 (2H, d, J=8.6, H-2,6 Ar); $8.62(1 \mathrm{H}, \mathrm{d}$, $\mathrm{J}=2.1, \mathrm{NH})$. Analysis: calc. for $\mathrm{C}_{18} \mathrm{H}_{19} \mathrm{~N}_{3} \mathrm{O}_{5}: \mathrm{C}$ 60.50, H 5.36, N 11.76. Found. C 60.44, H 5.33, N 11.60.

Methyl-2-(4-ethoxyphenyl)-1,

3 , 8-trioxodecahydro-1H-pyrrolo [3', 4':4, 5] pyrrolo [1, 2-a] pyrazine-9-carboxylate $3 \mathrm{~b}$. Yield $48 \%, \mathrm{mp}$

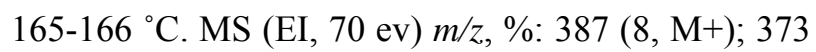
(21); 356 (5); 236 (67); 218 (100); 191 (25). ${ }^{1} \mathrm{H}$ NMR $\left(300 \mathrm{MHz}\right.$, ДMSO-d $\left.d_{6}\right) \delta: 1.39(3 \mathrm{H}, \mathrm{t}, \mathrm{J}=7.1$, $\left.\mathrm{CH}_{2} \underline{\mathrm{CH}}_{3}\right) ; 2.62-2.68(1 \mathrm{H}, \mathrm{m}, \mathrm{CH}) ; 2.82-2.88(1 \mathrm{H}, \mathrm{m}$, $\mathrm{CH})$; 3.21-3.25 $\left(2 \mathrm{H}, \mathrm{m}, \mathrm{CH}_{2} \mathrm{~N}\right) ; 3.51-3.56(1 \mathrm{H}, \mathrm{m}$, $\mathrm{CH})$; 3.57-3.62 (1H, m, $\left.\underline{\mathrm{CH}}_{2} \mathrm{NH}\right) ; 3.66\left(3 \mathrm{H}, \mathrm{s}, \mathrm{CH}_{3} \mathrm{O}\right)$; 3.71-3.73 (1H, m, $\left.\underline{\mathrm{CH}}_{2} \mathrm{NH}\right)$; 4.10-4.14 (2H, m, $\left.\mathrm{CH}_{2} \mathrm{O}\right)$; $4.37(1 \mathrm{H}, \mathrm{d}, \mathrm{J}=7.3, \mathrm{CH}) ; 7.01(2 \mathrm{H}, \mathrm{d}, \mathrm{J}=8.7 ; \mathrm{H}-3,5$ Ar); 7.09 (2H, d, J = 8.7, H-2,6 Ar); $8.39(1 \mathrm{H}, \mathrm{d}, \mathrm{J}=$
2.7, NH). Analysis: calc. for $\mathrm{C}_{19} \mathrm{H}_{21} \mathrm{~N}_{3} \mathrm{O}_{6}$ : C 58.91, $\mathrm{H}$ 5.46, N 10.85. Found. C 58.82, H 5.39, N 10.90.

2.3. General Method for the Synthesis of Methyl-1, 6-Dioxo-8-[(Arylamino) Carbonyl] - 1, 3, 4, 6, 7, 8 Hexahydro-2H-Pyrido [1, 2-a] Pyrazine-9-Carboxylates 4a-e

A mixture of $0.85 \mathrm{~g}(0.5 \mathrm{mmol})$ of 2-oxopiperazine 1 and $0.51 \mathrm{mmol}$ of the corresponding $\mathrm{N}$-arylmaleimide in $15 \mathrm{ml}$ of methanol with the addition of 2-3 drops of acetic acid is refluxed for 14 hours (control-TLC). The precipitate formed is filtered off after cooling, washed with ethanol. For removal of impurities addition products recrystallized twice from dimethylformamide. Received compounds 4a-e:

Methyl-1, 6-dioxo-8-[(phenylamino) carbonyl]-1, 3, 4, 6, 7, 8-hexahydro-2H-pyrido $\left[\begin{array}{ll}1, & 2-\mathrm{a}]\end{array}\right.$ pyrazine-9-carboxylate $4 \mathrm{a}$. Yield $52 \%$, mp $155-156^{\circ} \mathrm{C}$. MS (EI, 70 ev) m/z, \%: 343 (51, M+); 311 (100); 284 (23); 192 (42); 163 (41). ${ }^{1} \mathrm{H}$ NMR (300 MHz, ДMSO-d $\left.{ }_{6}\right) \delta: 2.42\left(2 \mathrm{H}, \mathrm{d}, \mathrm{J}=15.8, \mathrm{CH}_{2} \mathrm{CO}\right) ; 3.26-3.30$ $\left(2 \mathrm{H}, \mathrm{m}, \mathrm{CH}_{2} \mathrm{~N}\right) ; 3.46-3.50\left(1 \mathrm{H}, \mathrm{m}, \mathrm{CH}_{2} \mathrm{NH}\right) ; 3.62(3 \mathrm{H}$, $\left.\mathrm{s}, \mathrm{CH}_{3} \mathrm{O}\right) ; 3.72-3.74\left(1 \mathrm{H}, \mathrm{m}, \underline{\mathrm{CH}}_{2} \mathrm{NH}\right) ; 5.14(1 \mathrm{H}, \mathrm{br} \mathrm{s}$, $\mathrm{CH}) ; 7.05$ (2H, t, J=7.8; H-3,5 Ar); $7.12(2 \mathrm{H}, \mathrm{d}, \mathrm{J}=7.8$, $\mathrm{H}-2,6 \mathrm{Ar}) ; 7.18(2 \mathrm{H}, \mathrm{t}, \mathrm{J}=7.8 ; \mathrm{H}-3,5 \mathrm{Ar}) ; 8.74(1 \mathrm{H}, \mathrm{d}$, $\mathrm{J}=2.3, \mathrm{NH}) ; 9.48(1 \mathrm{H}, \mathrm{s}, \mathrm{NH}-\mathrm{Ar})$. Analysis: calc. for $\mathrm{C}_{17} \mathrm{H}_{17} \mathrm{~N}_{3} \mathrm{O}_{5}$ : C 59.47, H 4.99, N 12.24. Found. C 59.54, H 4.92, N 12.30 .

Methyl-8-\{[(4-methylphenyl) amino] carbonyl $\}-1$, 6-dioxo-1, 3, 4, 6, 7, 8-hexahydro-2H-pyrido [1, 2-a] pyrazine-9-carboxylate $4 \mathrm{~b}$. Yield $52 \%$, mp $152-153^{\circ} \mathrm{C}$. MS (EI, 70 ev) m/z, \%: 357 (48, M+); 325 (100); 298 (23); 192 (44); 163 (40). ${ }^{1} \mathrm{H}$ NMR (300 MHz, ДMSO-d $\left.d_{6}\right) \delta: 2.12\left(3 \mathrm{H}, \mathrm{s}, \mathrm{CH}_{3}\right) ; 2.26(2 \mathrm{H}, \mathrm{d}, \mathrm{J}=15.2$, $\left.\mathrm{CH}_{2} \mathrm{CO}\right)$; 3.34-3.39 $\left(2 \mathrm{H}, \mathrm{m}, \mathrm{CH}_{2} \mathrm{~N}\right)$; 3.43-3.48 $(1 \mathrm{H}, \mathrm{m}$, $\left.\mathrm{CH}_{2} \mathrm{NH}\right) ; 3.70\left(3 \mathrm{H}, \mathrm{s}, \mathrm{CH}_{3} \mathrm{O}\right) ; 3.73-3.75(1 \mathrm{H}, \mathrm{m}$, $\left.\underline{\mathrm{CH}}_{2} \mathrm{NH}\right) ; 5.28(1 \mathrm{H}$, br s, CH); $7.09(2 \mathrm{H}, \mathrm{d}, \mathrm{J}=8.4$; H-3,5 Ar); 7.28 (2H, d, J=8.4, H-2,6 Ar); $8.63(1 \mathrm{H}, \mathrm{d}$, $\mathrm{J}=2.7, \mathrm{NH}) ; 9.52(1 \mathrm{H}, \mathrm{s}, \mathrm{NH}-\mathrm{Ar})$. Analysis: calc. for $\mathrm{C}_{18} \mathrm{H}_{19} \mathrm{~N}_{3} \mathrm{O}_{5}: \mathrm{C}$ 60.50, H 5.36, N 11.76. Found. $\mathrm{C}$ 60.59, H 5.42, N 11.62. 


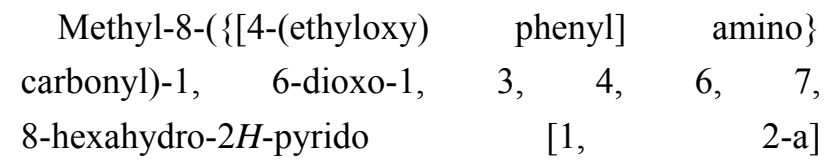
pyrazine-9-carboxylate 4c. Yield 42\%, mp 170-171 ${ }^{\circ} \mathrm{C}$. MS (EI, $\left.70 \mathrm{ev}\right) \mathrm{m} / \mathrm{z}, \%$ : 387 (58, M+); 355 (100); 328 (25); 192 (49); 163 (29). ${ }^{1} \mathrm{H}$ NMR (300 MHz, ДMSO-d 6 ) $\delta: 1.40\left(3 \mathrm{H}, \mathrm{t}, \mathrm{J}=7.3, \mathrm{CH}_{2} \mathrm{CH}_{3}\right) ; 2.20(2 \mathrm{H}$, $\left.\mathrm{d}, \mathrm{J}=15.8, \mathrm{CH}_{2} \mathrm{CO}\right) ; 3.32-3.36\left(2 \mathrm{H}, \mathrm{m}, \mathrm{CH}_{2} \mathrm{~N}\right)$; 3.51-3.58 (1H, m, $\left.\mathrm{CH}_{2} \mathrm{NH}\right) ; 3.71\left(3 \mathrm{H}, \mathrm{s}, \mathrm{CH}_{3} \mathrm{O}\right)$; 3.76-3.80 (1H, m, $\left.\underline{\mathrm{CH}}_{2} \mathrm{NH}\right)$; 4.02-4.08 (2H, m, $\left.\mathrm{CH}_{2} \mathrm{O}\right)$; $5.26(1 \mathrm{H}, \mathrm{br} \mathrm{s}, \mathrm{CH}) ; 6.98(2 \mathrm{H}, \mathrm{d}, \mathrm{J}=8.2 ; \mathrm{H}-3,5 \mathrm{Ar})$; $7.14(2 \mathrm{H}, \mathrm{d}, \mathrm{J}=8.2, \mathrm{H}-2,6 \mathrm{Ar}) ; 8.60(1 \mathrm{H}, \mathrm{d}, \mathrm{J}=2.1$, $\mathrm{NH}) ; 9.54(1 \mathrm{H}, \mathrm{s}, \mathrm{NH}-\mathrm{Ar})$. Analysis: calc. for $\mathrm{C}_{19} \mathrm{H}_{21} \mathrm{~N}_{3} \mathrm{O}_{6}$ : C 58.91, H 5.46, N 10.85. Found. $\mathrm{C}$ 58.80, H 5.52, N 10.75 .

Methyl-8-\{[(4-chlorophenyl) amino] carbonyl $\}-1$, 6-dioxo-1, 3, 4, 6, 7, 8-hexahydro- $2 \mathrm{H}$-pyrido [1, 2-a] pyrazine-9-carboxylate $4 \mathrm{~d}$. Yield 45\%, mp 163-164

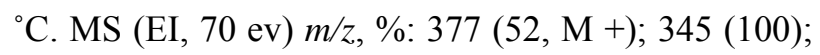
318 (21); 192 (37); 163 (25). ${ }^{1} \mathrm{H}$ NMR (300 MHz, ДMSO-d $\left.)_{6}\right) \delta: 2.65\left(2 \mathrm{H}, \mathrm{d}, \mathrm{J}=16.1, \mathrm{CH}_{2} \mathrm{CO}\right)$; 3.31-3.36 (2H, m, $\left.\mathrm{CH}_{2} \mathrm{~N}\right)$; 3.39-3.43 (1H, m, $\left.\mathrm{CH}_{2} \mathrm{NH}\right)$; 3.66-3.70 (1H, m, $\left.\underline{\mathrm{CH}}_{2} \mathrm{NH}\right) ; 3.72\left(3 \mathrm{H}, \mathrm{s}, \mathrm{CH}_{3} \mathrm{O}\right) ; 5.31$ $(1 \mathrm{H}, \mathrm{br} \mathrm{s}, \mathrm{CH}) ; 7.38(2 \mathrm{H}, \mathrm{d}, \mathrm{J}=8.7$; H-3,5 Ar); 7.52 $(2 \mathrm{H}, \mathrm{d}, \mathrm{J}=8.7, \mathrm{H}-2,6 \mathrm{Ar}) ; 8.78(1 \mathrm{H}, \mathrm{d}, \mathrm{J}=3.6, \mathrm{NH})$; $9.58(1 \mathrm{H}, \quad \mathrm{s}, \quad \mathrm{NH}-\mathrm{Ar})$. Analysis: calc. for $\mathrm{C}_{17} \mathrm{H}_{16} \mathrm{ClN}_{3} \mathrm{O}_{5}$ : C 54.05, H 4.27, N 11.11. Found. C 54.00, H 4.38, N 11.22.

Methyl-8-\{[(2, 4-dichlorophenyl) amino $]$ carbonyl\}-1, 6-dioxo-1, 3, 4, 6, 7, 8-hexahydro-2H-pyrido [1, 2-a] pyrazine-9-carboxylate 4e. Yield $47 \%$, mp $200-202^{\circ} \mathrm{C}$. MS (EI, 70 ev) $\mathrm{m} / \mathrm{z}$, \%: 412 (8, M +); 380 (100); 353 (17); 192 (46); 163 (29). ${ }^{1} \mathrm{H}$ NMR (300 MHz, ДMSO-d $\left.)_{6}\right) \delta: 2.63(2 \mathrm{H}, \mathrm{d}, \mathrm{J}=$ 16.1, $\left.\mathrm{CH}_{2} \mathrm{CO}\right)$; 3.30-3.34 (2H, m, $\left.\mathrm{CH}_{2} \mathrm{~N}\right)$; 3.40-3.45 $\left(1 \mathrm{H}, \mathrm{m}, \underline{\mathrm{CH}_{2}} \mathrm{NH}\right) ; 3.66-3.71\left(1 \mathrm{H}, \mathrm{m}, \underline{\mathrm{CH}_{2}} \mathrm{NH}\right) ; 3.74$ $\left(3 \mathrm{H}, \mathrm{s}, \mathrm{CH}_{3} \mathrm{O}\right) ; 5.34(1 \mathrm{H}$, br s, $\mathrm{CH}) ; 7.32(1 \mathrm{H}, \mathrm{s}, \mathrm{H}-3$ $\mathrm{Ar}) ; 7.36(1 \mathrm{H}, \mathrm{d}, \mathrm{J}=8.7$; H-5 $\mathrm{Ar}) ; 7.48(1 \mathrm{H}, \mathrm{d}, \mathrm{J}=8.7$, H-6 Ar); $8.78(1 \mathrm{H}, \mathrm{d}, \mathrm{J}=3.6, \mathrm{NH}) ; 9.58(1 \mathrm{H}, \mathrm{s}$, NH-Ar). Analysis: calc. for $\mathrm{C}_{17} \mathrm{H}_{15} \mathrm{Cl}_{2} \mathrm{~N}_{3} \mathrm{O}_{5}$ : C 49.53, $\mathrm{H}$ 3.67, N 10.19. Found. C 49.65, H 3.60, N 10.29.

\section{Results and Discussion}

\subsection{Synthesis and Characterization}

We have studied the interaction of methyl (3-oxo-piperazine-2-ylidene) acetate 1 (prepared by reacting ethylenediamine with dimethyl acetylenedicarboxylate [10]) with $\mathrm{N}$-arylmaleimides 2 . It is known that $N$-arylmaleimides are dipolarophiles [11], at the same time, under the action of nucleophiles occurs recyclization maleimide fragment [12]. It was reported earlier [10] that methyl (3-oxopiperazin-2-ylidene) acetate 1 in an acidic medium can be converted to the tautomeric form methyl (3-oxo-3, 4, 5, 6-tetrahydropyrazine-2-yl) acetate 1' (Fig. 1). We hypothesized that in the reactions of simultaneous involving nitrogen atoms $\mathrm{N}$ (4) and the side chain depending on the condition, the this heterocycle may act as the $C-N$-dinucleophile, being in the form of the enamine 1 , and as the 1 , 3-dipole- when transition to form imine 1'.

In this work, we have found that when the reaction of the starting compounds by refluxing in a mixture of methanol and acetic acid (in the ratio 1:1) arylmaleimides 2 attached to the imine $1^{\prime}$ as a dipole to form a new heterocyclic system -methyl-2-aryl-1, 3, 8-trioxodecahydro-1H-pyrrolo [3', 4':4, 5] pyrrolo [1, 2-a] pyrazine-9-carboxylates 3a, b (Fig. 1). With a deficiency of acid (the use of catalytic amounts) starting compound is reacted with an arylmaleimide 2 mainly as enamine 1 , wherein the maleimide recyclization occurs leading to not previously described methyl-1, 6-dioxo-8-[(arylamino) carbonyl]-1, 3, 4, 6, 7, 8-hexahydro-2H-pyrido [1,2-a] pyrazine-9-carboxylates 4a-e (Fig. 1).

At carrying out the reaction under different conditions (refluxing the reactants in toluene without catalyst, in the presence of acidic catalysts, in butanol-1-and stirring at room temperature in acetic acid) is formed inseparable mixture of products 3 and 4. The structure of the obtained for the first time chemical compounds 3 and 4 is uniquely proved by ${ }^{1} \mathrm{H}$ 


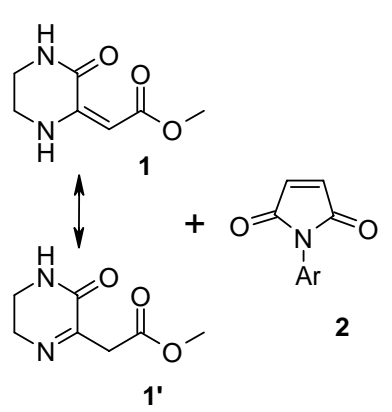

$3 \mathrm{Ar}=4-\mathrm{CH}_{3}-\mathrm{C}_{6} \mathrm{H}_{4}(\mathrm{a}), 4-\mathrm{C}_{2} \mathrm{H}_{5} \mathrm{O}-\mathrm{C}_{6} \mathrm{H}_{4}$ (b);

$4 \mathrm{Ar}=\mathrm{C}_{6} \mathrm{H}_{5}(\mathrm{a}), 4-\mathrm{CH}_{3}-\mathrm{C}_{6} \mathrm{H}_{4}$ (b), 4- $\mathrm{C}_{2} \mathrm{H}_{5} \mathrm{O}-\mathrm{C}_{6} \mathrm{H}_{4}$ (c); 4-Cl- $\mathrm{C}_{6} \mathrm{H}_{4}$ (d), 2,4-diCl- $\mathrm{C}_{6} \mathrm{H}_{4}$ (e)

Fig. 1 The interaction of methyl

(3-oxopiperazin-2-ylidene) acetate with an

$N$-arylmaleimides.

NMR spectroscopy and mass spectrometry.

\section{Conclusions}

It is established that methyl (3-oxo-piperazine-2-ylidene) acetate is reacted with $\mathrm{N}$-arylmaleimides in boiling methanol in the presence of catalytic amounts of acetic acid as the $C$ - $N$-dinucleophile. An excess of acid occurs 1,3-dipolar cycloaddition of the $N$-arylmaleimides toward tautomeric form methyl (3-oxo-piperazine-2-ylidene) acetate-methyl (3-oxo-3,4,5,6-tetrahydropyrazine-2-yl) acetate.

\section{Acknowledgments}

The results obtained in the framework of the work on the RF Government Resolution № 218 contract N 02.G25.31.0007 supported by the Ministry of Education and Science of the Russian Federation.

\section{References}

[1] Risi, C. D., Pela, M., Pollini, G-P., Trapella, C. and Zanirato, V. 2010. "Mastering Chiral Substituted 2-Oxopiperazines." Tetrahedron: Asymmetry 21: 255-74.

[2] Qian, W., Chen, J. J., Human, J., Aya, T., Zhu, J. and Biswas, K. et al. 2012. "Discovery of
Dehydro-Oxopiperazine Acetamides as Novel Bradykinin B1 Receptor Antagonists with Enhanced in Vitro Potency, Bioorg." Med. Chem. Lett. 22: 1061-7.

[3] Trost, B. M. and Dong, G. 2006. "New Class of Nucleophiles for Palladium-Catalyzed Asymmetric Allylic Alkylation." Total synthesis of agelastatin A, J. Am. Chem. Soc. 128: 6054-5.

[4] Trost, B. M., Cramer, N. and Bernsmann, H. 2007. "Concise Total Synthesis of ( \pm -Marcfortine B." J. Am. Chem. Soc. 129: 3086-7.

[5] Jacquot, D. E. N., Hoffmann, H., Polborn, K. and Lindel, T. 2002. "Synthesis of the Dipyrrolopyrazinone Core of Dibromophakellstatin and Related Marine Alkaloids." Tetrahedron Lett. 43: 3699-702.

[6] Jana, A. K., Das, S. K. and Panda, G. 2012. “An Efficient Entry to highly Substituted Chiral 2-Oxopiperazines from $\alpha$-Amino Acids via Iodocyclization." Tetrahedr. 68: 10114-21.

[7] Kawahara, N., Nakajima, T., Itoh, T. and Ogura, H. 1983. “A Synthesis of Pyrido [1.2-a] Qunoxalines and Pyrido [1.2-a] Pyrazines.” Heterocycl 20: 1721-5.

[8] Bernotas, R. C. 2004. "A Short, Novel Approach to 2, 3, 4a, 5-Tetrahydro-1H-Pyrazino [1, 2-a] Quinoline-4, 6-Diones." Synlett 12: 2165-6.

[9] Medvedeva, S. M., Stashina, G. A., Firgang, S. I., Malikova, E. S., Krysin, M. Yu. and Shikhaliev, K. S. 2014. "Azomethine Ylides Derived from Alkyl (3-oxopiperazin-2-yl) Acetates in 1, 3-Dipolar Cycloaddition Reactions with N-Arylmaleimides." Chem. Heterocycl. Compd. 50: 537-43.

[10] Iwanami, Y. 1971. "The Reaction of Acetylenecarboxylic Acid with Amines. XV. Reaffirmation of the Enamine Structure Facilitated by Intramolecular Hydrogen Bonding Common to the Reaction Products." Bull. Chem. Soc. Jpn. 44: 1311-3.

[11] Garner, P. and Kaniskan, U. 2005. "Synthesis of Highly Functionalized Pyrrolidines via a Mild One-Pot, Three-Component 1, 3-Dipolar Cycloaddition Process." J. Org. Chem. 70: 10868-71.

[12] Rudenko, R. V., Komykhov, S. A., Desenko, S. M., Sen'ko, Y.V., Shishkin, O. V. and Konovalova, I. S. et al. 2011. "A Comprehensive Study of the Heterocyclizations of N-Arylmaleimides and 6-Aminouracils." Synthesis 19: 3161-7. 